\title{
Acupuntura para o tratamento da dismenorreia primária: Principais pontos
}

\author{
Acupuncture for the treatment of primary dysmenorhea: Main points \\ Acupuntura para el tratamiento de la dismenorea primaria: Principales puntos
}

Recebido: 06/11/2021 | Revisado: 12/11/2021 | Aceito: 17/11/2021 | Publicado: 27/11/2021

Pablo Rafael De Negreiros Marques ORCID: https://orcid.org/0000-0002-5915-5336 Faculdade Facottur, Brasil E-mail: pablo.marques1977@gmail.com

Tayanna Alves De Almeida ORCID: https://orcid.org/0000-0002-3005-024X Faculdade Facottur, Brasil E-mail: tayannaalmeida@hotmail.com

Mateus Porfirio Rodrigues ORCID: https://orcid.org/0000-0002-6747-4898 Faculdade Facottur, Brasil

E-mail: mateusporfirio6@gmail.com

Ingrid Larissa da Silva Laurindo ORCID: https://orcid.org/0000-0001-7658-2455 Faculdade Facottur, Brasil

E-mail: larissasilvalaurindo@gmail.com

Eudson José Santos do Monte ORCID: https://orcid.org/0000-0002-1871-6619 Faculdade Facottur, Brasil E-mail: eudson41@gmail.com

Amanda Fonseca do Nascimento ORCID: https://orcid.org/0000-0001-7804-671X Faculdade Facottur, Brasil

E-mail: amandas2nascimento@gmail.com

Flávia Carolina Lasalvia da Silva ORCID: https://orcid.org/0000-0003-3441-3201 Faculdade Facottur, Brasil E-mail: flaviaclasalvia@gmail.com

Harrison Euller Vasconcelos Queiroz ORCID: https://orcid.org/0000-0001-7906-2981 Faculdade Facottur, Brasil E-mail: harrisoneuller@gmail.com Sidney Queiroz de Sá Leitão ORCID: https://orcid.org/0000-0001-8627-2688 Faculdade Facottur, Brasil

E-mail: sidneyleitao@hotmail.com

José Carlos Nogueira Nóbrega Júnior ORCID: https://orcid.org/0000-0003-3908-9260 Faculdade Facottur, Brasil E-mail: c10carlo@gmail.com

\begin{abstract}
Resumo
Objetivo: Descrever os principais pontos de acupuntura utilizados no tratamento da dismenorreia primária. Método: Trata-se de uma revisão de literatura narrativa, onde foram utilizados ensaios clínicos, com os seguintes descritores: "Acupuncture", "Acupuncture Points", "Chinese Medicine" e "Primary Dysmenorrhea". As buscas ocorreram nas bases de dados da SciELO, PubMed, LILACS, PEDro, Portal de Periódicos CAPES, ScienceDirect e Cochrane no período de 2011 a 2021, com artigos selecionados entre janeiro 2016 a dezembro 2021, em qualquer idioma, que apresentassem em seu objetivo de pesquisa descrever os principais pontos de acupuntura utilizados no tratamento da dismenorreia primária. Foram considerados inábeis artigos duplicados e pagos, de revisão, meta-análises e mulheres que apresentassem patologias associadas. Resultados: Foram identificados 334 artigos, dos quais 5 preencheram os critérios de inclusão, abrangendo um total de 348 pacientes com diagnostico de dismenorreia primaria. $\mathrm{O}$ uso terapêutico do baço 6 (BP-6) foi avaliado em três das cincos publicações selecionadas, atendendo a diversos objetivos. O desfecho primário mais avaliado foi a intensidade da dor menstrual. Conclusão: Conclui-se que a acupuntura pode ser usada como um método eficaz e seguro no tratamento em mulheres com dismenorreia primaria.
\end{abstract}

Palavras-chave: Acupuntura; Pontos de acupuntura; Medicina chinesa; Dismenorreia. 


\begin{abstract}
Aim: To describe the main acupuncture points used in the treatment of primary dysmenorrhea. Method: This is a review of narrative literature, where clinical trials were used, with the following descriptors: "Acupuncture", "Acupuncture Points", "Chinese Medicine" e "Primary Dysmenorrhea". The searches took place in the SciELO, PubMed, LILACS, PEDro, CAPES Journal Portal, ScienceDirect and Cochrane databases, in the period from 2011 to 2021, with articles selected between January 2016 to December 2021, in any language, that presented in their research objective to describe the main acupuncture points used in the treatment of primary dysmenorrhea. Duplicate and paid review articles were considered unskillful, meta-analyses and women with associated pathologies. Results: 334 articles were identified, of which 5 met the inclusion criteria, covering a total of 348 patients diagnosed with primary dysmenorrhea. The therapeutic use of spleen 6 (BP-6) was evaluated in three of the five selected publications, meeting several objectives. The most evaluated primary outcome was the intensity of menstrual pain. Conclusion: It is concluded that acupuncture can be used as an effective and safe method to treat women with primary dysmenorrhea.
\end{abstract}

Keywords: Acupuncture; Acupuncture points; Chinese medicine; Dysmenorrhea.

\title{
Resumen
}

Objetivo: Describir los principales puntos de acupuntura utilizados en el tratamiento de la dismenorrea primaria. Método: Esta es una revisión de la literatura narrativa, donde se utilizaron ensayos clínicos, con los siguientes descriptores: Acupuntura, Puntos de acupuntura, Medicina china, Dismenorrea primaria. Las búsquedas se realizaron en las bases de datos SciELO, PubMed, LILACS, PEDro, CAPES Journal Portal, ScienceDirect y Cochrane de 2011 a 2021, con artículos seleccionados de enero de 2016 a diciembre de 2021, en cualquier idioma, que presentó en su objetivo de investigación para describir los principales puntos de acupuntura utilizados en el tratamiento de la dismenorrea primaria. Los artículos duplicados y pagados se consideraron poco hábiles, revisión, metaanálisis y mujeres con patologías asociadas. Resultados: se identificaron 84 artículos, de los cuales 5 cumplieron con los criterios de inclusión, cubriendo un total de 348 pacientes diagnosticados de dismenorrea primaria. Se evaluó el uso terapéutico del bazo 6 (BP-6) en tres de las cinco publicaciones seleccionadas, cumpliendo varios objetivos. El resultado primario más evaluado fue la intensidad del dolor menstrual. Conclusión: Se concluye que la acupuntura puede utilizarse como un método eficaz y seguro para tratar a mujeres con dismenorrea primaria.

Palabras clave: Acupuntura; Puntos de acupuntura; Medicina china; Dismenorrea.

\section{Introdução}

A dismenorreia é uma dor pélvica ou abdominal inferior, cíclica ou recorrente, associada à menstruação. É a queixa ginecológica mais comum em mulheres jovens, com uma prevalência significativamente alta. De acordo com a intensidade dos sintomas, é também uma causa importante de absentismo escolar ou laboral. Cerca de 50 a $70 \%$ das mulheres apresentam sintomas de dismenorreia em algum momento da vida, sendo que aproximadamente $10 \%$ se tornam incapazes de desenvolver suas atividades habituais (Xie, et al., 2011). Vários tratamentos são propostos para a dismenorreia e incluem o uso de antiinflamatórios não hormonais (AINH), anticoncepcionais orais, vitaminas e agentes tocolíticos (Hu, et al., 2019).

Os terapeutas especializados em Medicina Chinesa trabalham com a energia Qi e seus movimentos. Os pontos de Acupuntura possuem tanto um componente anatômico como energético. Nesse sentido, o ponto é, por assim dizer, “vivo". Eles são ligados à rede de canais de energia (meridianos) e permanecem em comunicação alternada com o interior e exterior do corpo. Para desenvolver por completo o possível potencial de um tratamento de Acupuntura, é importante estimular o ponto de maneira adequada, por exemplo, por meio de técnicas auxiliares de manipulação (Ma, et al., 2013).

A Acupuntura atua sobre as patologias por meio das agulhas. Consiste em inserir uma agulha metálica de corpo longo e ponta fina em determinados lugares (pontos), aplicando certos meios de manipulação para produzir sensações no paciente, intumescimento, distensão e sensação de peso (De Qi), com a finalidade de curar uma enfermidade (Liu, et al., 2011).

É fundamental saber que os cinco elementos, nos quais se baseiam a filosofia chinesa, derivam de cinco fontes de vida. O sistema é polarizado: O positivo e o negativo (Teoria do Yin Yang). De acordo com a Medicina Chinesa (MC), o tratamento através da Acupuntura tem como objetivo a normalização dos órgãos doentes e o equilíbrio desses elementos por meio de um suporte funcional que exerce efeito terapêutico (Hang, et al., 2015).

Levando em consideração que o aparelho genital feminino tem ligação direta com o Vaso Concepção (Ren Mai), Vaso Penetrador (Chong Mai) e o Rim (Shen), promovem a força para os Vasos Extraordinários permitirem a Menstruação, 
Concepção e Gravidez. Leva-se em consideração os órgãos envolvidos diretamente com o Sangue: Coração (Xin) que regula a circulação sanguínea, Fígado (Gan) que armazena o Sangue e o Baço (Pi) que produz e controla o Sangue, na compreensão da Menstruação e no tratamento dos desconfortos que acontecem com a Mulher, durante os ciclos menstruais (Chen, et al., 2011).

A etiologia e etiopatogenia da DP decorre, principalmente, do fluxo obstruído do Qi e do sangue que pode ser causado pelos seguintes fatores: estagnação de Qi e sangue causam distúrbios emocionais, produzindo dor durante a menstruação; exposição a lugares frios e úmidos, ingestão excessiva de alimentos frios e crus favorecem ao congelamento e fluxo do sangue, causando dor; Qi e sangue inadequados, decorrente de patologias crônicas ou constituição debilitada, gerando também a sensação de dor (Liu, et al., 2014).

A Acupuntura tem sido usada a milhares de anos no tratamento de doenças e na prevenção e manutenção da saúde da mulher, desta forma esse trabalho visa descrever os principais pontos de acupuntura para o tratamento da dismenorreia primária.

\section{Metodologia}

O presente estudo se trata de uma revisão de literatura, elaborada no período de abril a julho de 2021, com o propósito de trazer pesquisas mais atualizadas, a fim de abordar os resultados das análises de autores variados, referente a acupuntura em mulheres que sofrem com dismenorreia primária.

Para a realização da seleção dos artigos, a avaliação foi feita por dois pesquisadores de forma independente, a qual posteriormente estes se reuniram para verificação de possíveis divergências de opiniões e, por fim, a realização de um consenso.

Com relação a execução do estudo, teve-se como base: criar a pergunta principal, realizar uma busca de autores que trabalham com o tema proposto, selecionar os melhores artigos, realizar a extração dos dados e avaliar a qualidade metodológica. A causa que direciona este estudo é: Descrever os principais pontos de acupuntura para o tratamento da dismenorreia primária.

Inicialmente, realizou-se uma busca na plataforma dos Descritores em Ciências da Saúde (DeCS), sendo assim definido os seguintes descritores: Acupuncture, Acupuncture Points, Chinese Medicine e Dysmenorrhea, que foram selecionados para a busca nas bases de dados: Scientific Electronic Library Online (SciELO), Pubmed, Literatura LatinoAmericana e do Caribe em Ciências da Saúde (LILACS), Phyiotherapy Evidence Database (PEDro), Portal de Periódicos CAPES, ScienceDirect e Cochrane. Durante as buscas dos descritores foram utilizados de formas variadas entre si e foram utilizados os operadores booleanos "AND" ou "OR", em seguida foi realizada uma análise crítica dos estudos incluídos, assim sendo realizada a discussão dos resultados.

Para critérios de inclusão, foram selecionados artigos com o corte temporal dos últimos onze anos (2011 a 2021); artigos que utilizassem os protocolos aplicados a patologia de forma detalhada e que não fugissem da temática escolhida, estudos em qualquer idioma e ensaios clínicos. Foram excluídos estudos que embora tivessem os mesmos descritores pesquisados não abordavam o tema proposto, estudos com administração de fármacos, literatura cinzenta, trabalhos que abordassem a dismenorreia secundária, quaisquer tipos de estudos que não fossem ensaio clínico, trabalhos em que o tema proposto não estivesse de forma clara e objetiva nas suas metodologias de aplicação.

Assim sendo, seguiu-se com a inspeção de fundamentação teórica e a observação das características gerais dos estudos. Foi realizado o parecer de toda metodologia aplicada, discussão, resultados obtidos e análise descritiva de cada um dos textos. Técnicas qualitativas e/ou quantitativas específicas de tratamento de dados para analisar a produção científica não foram utilizadas. 


\section{Resultados}

Inicialmente foi realizada a busca nas bases de dados supracitadas, onde foram encontrados 334 artigos, em seguida realizou-se a filtragem inicial por título e resumo dos artigos encontrados e aplicação dos critérios de inclusão e exclusão, na última etapa foi realizado a leitura nos completa dos estudos. Sendo assim, esta revisão foi constituída por 5 artigos. As Etapas do processo de busca são descritas no fluxograma abaixo (Figura 1).

Figura 1: Fluxograma da busca dos artigos.

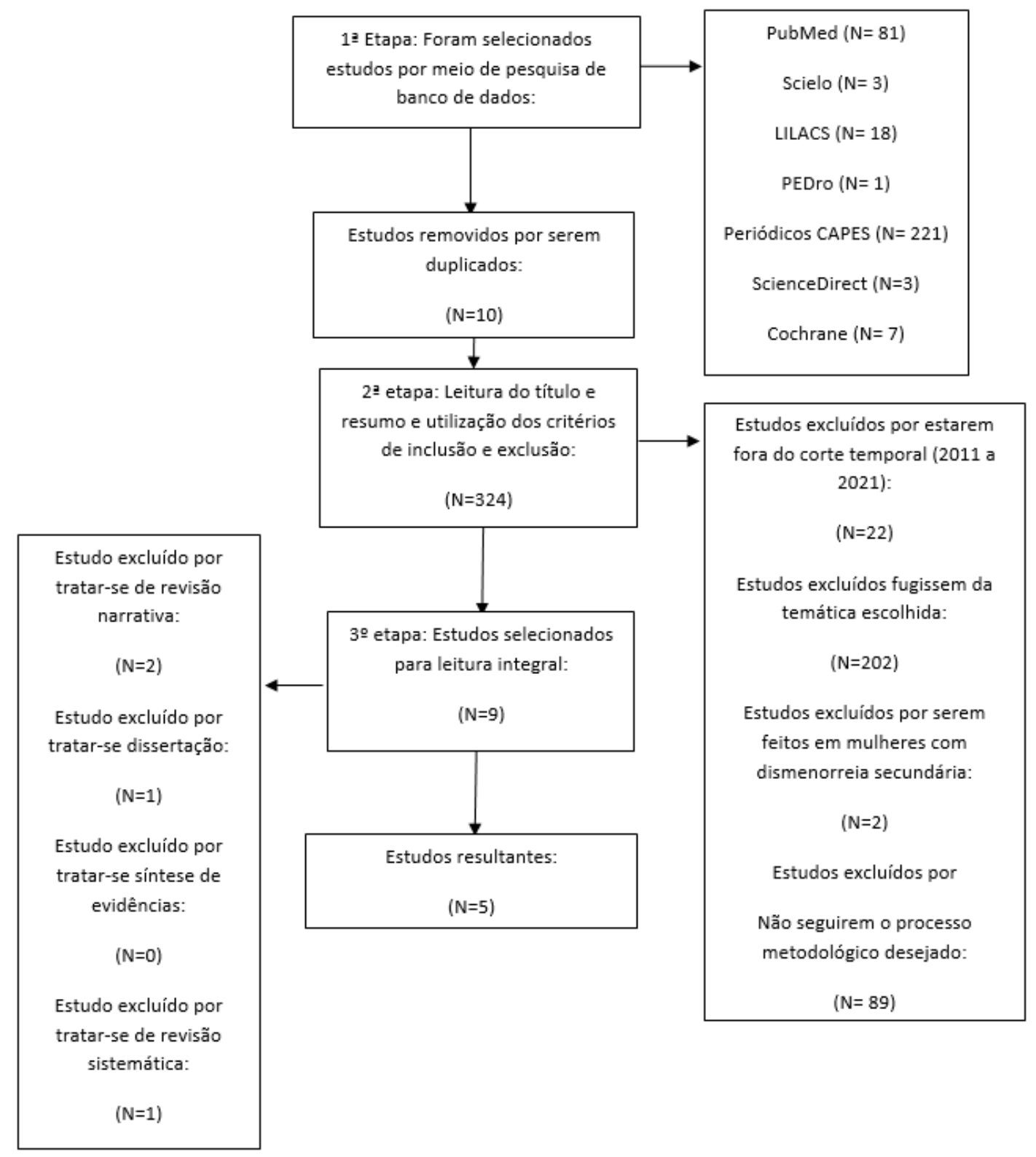

Fonte: Autores (2021).

A Tabela 1 mostra a análise das referências, permitindo assim que houvesse uma melhor organização na comparação entre os estudos. Nela, estão descritos o autor, ano, tipo de estudo, pontos utilizados, título, objetivos e desfechos. 
Tabela 1: Características gerais dos estudos selecionados.

\begin{tabular}{|c|c|c|c|c|c|}
\hline Autor/Ano & $\begin{array}{l}\text { Tipo de } \\
\text { estudo }\end{array}$ & $\begin{array}{c}\text { Pontos } \\
\text { utilizados }\end{array}$ & Título do estudo & Objetivo & Desfecho \\
\hline $\begin{array}{l}\text { Shetty B., } \\
\text { et. al., } 2018\end{array}$ & $\begin{array}{c}\text { Ensaio } \\
\text { Clínico } \\
\text { Randomizad } \\
\text { o }\end{array}$ & $\begin{array}{l}\text { R-3, BP-8, } \\
\text { E-25, E-29, } \\
\text { E-30, E-36, } \\
\text { VC-4, VC- } \\
\text { 6, B-62, C- } \\
\text { 7, IG-4 e } \\
\text { PC-6 }\end{array}$ & $\begin{array}{l}\text { Efficacy of } \\
\text { Acupuncture in the } \\
\text { Management of } \\
\text { Primary } \\
\text { Dysmenorrhoea: A } \\
\text { Randomized } \\
\text { Controlled Trial }\end{array}$ & $\begin{array}{l}\text { Avaliar a eficácia } \\
\text { da acupuntura em } \\
\text { mulheres com } \\
\text { dismenorreia } \\
\text { primaria, na } \\
\text { redução dos } \\
\text { sintomas. }\end{array}$ & $\begin{array}{c}\text { Este estudo mostrou uma redução } \\
\text { significativa em todas as variáveis, como } \\
\text { pontuação na escala visual analógica para } \\
\text { dor, cólicas menstruais, dor de cabeça, } \\
\text { tontura, diarreia, desmaio, alterações de } \\
\text { humor, cansaço, náusea, vômito no grupo de } \\
\text { estudo em comparação com o grupo controle. }\end{array}$ \\
\hline $\begin{array}{l}\text { Wang, et. } \\
\text { al., } 2019\end{array}$ & $\begin{array}{c}\text { Ensaio } \\
\text { Clínico } \\
\text { Randomizad } \\
\text { o }\end{array}$ & $\begin{array}{l}\text { B-54, E-28, } \\
\text { IG-4, F-3, } \\
\text { B-32, BP- } \\
10, \text { B-20, E- } \\
36\end{array}$ & $\begin{array}{c}\text { Effect of an } \\
\text { Acupuncture } \\
\text { Technique of } \\
\text { Penetrating through } \\
\text { Zhibian (BL54) to } \\
\text { Shuidao (ST28) } \\
\text { with Long Needle } \\
\text { for Pain Relief in } \\
\text { Patients with } \\
\text { Primary } \\
\text { Dysmenorrhea: A } \\
\text { Randomized } \\
\text { Controlled Trial }\end{array}$ & $\begin{array}{l}\text { Analisar o método } \\
\text { de acupuntura com } \\
\text { agulha longa para o } \\
\text { alívio da dor em } \\
\text { pacientes com } \\
\text { dismenorreia } \\
\text { primaria. }\end{array}$ & $\begin{array}{l}\text { Este estudo mostrou que o tratamento por } 3 \\
\text { ciclos menstruais, utilizando agulhamento } \\
\text { profundo nos pontos B-54 e E- } 28 \text { foi mais } \\
\text { eficaz do que o uso da medicação Ibuprofeno } \\
\text { na redução dos sintomas da dor menstrual. }\end{array}$ \\
\hline $\begin{array}{l}\text { Sun, et. al., } \\
\quad 2018\end{array}$ & $\begin{array}{l}\text { Ensaio } \\
\text { clínico } \\
\text { controlado e } \\
\text { randomizado }\end{array}$ & $\begin{array}{c}\text { BP-6, BP-8 } \\
\text { e BP-9 }\end{array}$ & $\begin{array}{c}\text { Acupuncture } \\
\text { Treatment of } \\
\text { Primary } \\
\text { Dysmenorrhea by } \\
\text { Needling Acupoints } \\
\text { of the Spleen } \\
\text { Meridian with } \\
\text { Positive } \\
\text { Reactions : A } \\
\text { Randomized } \\
\text { Controlled Clinical } \\
\text { Trial }\end{array}$ & $\begin{array}{c}\text { Observar se o } \\
\text { agulhamento de } \\
\text { pontos de gatilho } \\
\text { no trajeto do } \\
\text { meridiano do baço } \\
\text { pode ter um melhor } \\
\text { efeito terapêutico } \\
\text { do que realizar } \\
\text { acupuntura dos } \\
\text { pontos do Baço } \\
\text { (BP-6, BP-8 e BP- } \\
\text { 9) em pacientes } \\
\text { com DP. }\end{array}$ & $\begin{array}{l}\text { Após o tratamento, as pontuações para as } \\
\text { escalas visual analógica e de sintomas } \\
\text { menstruais diminuíram significativamente em } \\
\text { ambos os grupos de controle e observação, } \\
\text { sugerindo um alívio acentuado da dor e } \\
\text { desconforto. }\end{array}$ \\
\hline $\begin{array}{l}\text { Zhao, et. } \\
\text { al., } 2017\end{array}$ & $\begin{array}{l}\text { Ensaio } \\
\text { clínico } \\
\text { multicêntrico } \\
\text { randomizado } \\
\text { e controlado }\end{array}$ & BP-6 & $\begin{array}{l}\text { Influence of De Qi } \\
\text { on the immediate } \\
\text { analgesic effect of } \\
\text { BP-6 acupuncture in } \\
\text { patients with } \\
\text { primary } \\
\text { dysmenorrhoea and } \\
\text { cold and dampness } \\
\text { stagnation: a } \\
\text { multicentre } \\
\text { randomised } \\
\text { controlled trial }\end{array}$ & $\begin{array}{c}\text { Investigar a } \\
\text { contribuição do De } \\
\text { Qi para o efeito } \\
\text { analgésico imediato } \\
\text { da acupuntura em } \\
\text { pacientes com DP e } \\
\text { o diagnóstico } \\
\text { específico da } \\
\text { medicina } \\
\text { tradicional chinesa } \\
\text { estagnação de frio e } \\
\text { umidade. }\end{array}$ & $\begin{array}{c}\text { Este estudo não mostrou diferença } \\
\text { significativa nos escores de escala visual } \\
\text { analógica de dor em pacientes com } \\
\text { dismenorreia primaria e estagnação de frio e } \\
\text { umidade logo após a acupuntura do BP-6. Os } \\
\text { pacientes que tiveram a sensação do De Qi } \\
\text { demonstraram maiores reduções no escore de } \\
\text { dor em relação aqueles que não tiveram a } \\
\text { sensação do De Qi, sugerindo maiores efeitos } \\
\text { analgésicos. }\end{array}$ \\
\hline $\begin{array}{l}\text { Wang, et. } \\
\text { al., } 2018\end{array}$ & $\begin{array}{c}\text { Ensaio } \\
\text { clínico } \\
\text { randomizado }\end{array}$ & BP-6 & $\begin{array}{l}\text { Analgesic Effect of } \\
\text { Deqi Induced by } \\
\text { Needling at } \\
\text { Sanyinjiao (BP-6) } \\
\text { Acupoint on } \\
\text { Primary } \\
\text { Dysmenorrheal } \\
\text { Patients with Cold } \\
\text { Damp Stagnation } \\
\text { Syndrome }\end{array}$ & $\begin{array}{l}\text { Observar o efeito } \\
\text { analgésico e o } \\
\text { processo de } \\
\text { manipulação da } \\
\text { agulha para indução } \\
\text { do De Qi por } \\
\text { agulhamento no } \\
\text { Sanyinjiso (BP-6) } \\
\text { em pacientes com } \\
\text { DP e com síndrome } \\
\text { de estagnação a frio } \\
\text { e umidade. }\end{array}$ & $\begin{array}{l}\text { O método de intervenção com agulha grossa, } \\
\text { inserção profunda e manipulação é mais fácil } \\
\text { na indução do De Qi do que o método de } \\
\text { agulha fina, inserção rasa e nenhuma } \\
\text { manipulação. O efeito analgésico do De Qi é } \\
\text { melhor do que o não De Qi em pacientes com } \\
\text { DP e síndrome de estagnação frio umidade. }\end{array}$ \\
\hline
\end{tabular}

Nota: $\mathrm{B}=$ Bexiga $; \mathrm{E}=$ Estômago; $\mathrm{BP}=\mathrm{Baço} ; \mathrm{R}=\mathrm{Rim} ; \mathrm{VC}=$ Vaso Concepção; $\mathrm{C}=$ Coração; $\mathrm{IG}=$ Intestino Grosso; PC = Pericárdio Fonte: Autores (2021). 


\section{Discussão}

Nos artigos selecionados Shetty B. et al., (2018), Wang, et al., (2019), Sun, et al., (2018), Zhao, et al., (2017) e Wang, et al., (2018) foram avaliadas 348 mulheres que apresentavam queixas de dismenorreia primaria de leve a severa, com pontuação significativa na escala visual analógica de dor (EVA). Na diferenciação das síndromes, observou-se quadros de estagnação de frio e umidade (CDSS), estagnação de Qi e estase de sangue. Todos os estudos tiveram o período de intervenção de três ciclos menstruais e todas as pacientes com fases regulares de menstruação.

Os resultados mostraram que a maior parte dos artigos publicados (56,5\%) no período analisado, ou seja, 2016 a 2020 foram realizadas nos últimos três anos. Outro ponto a destacar no período analisado é o aumento de estudos clínicos $(67,6 \%)$ com relação às revisões de literatura $(32,4 \%)$ sobre o tema.

O estudo de Shetty B. et al., (2018) avaliou a diminuição de diversos sintomas em mulheres com DP, utilizando 12 pontos regulares da acupuntura, sendo eles: rim 3 (R-3), baço 8 (BP-8), estômago 25 (E-25), estômago 29 (E-29), estômago 30 (E-30), estômago 36 (E-36), vaso concepção 4 (VC-4), vaso concepção 6 (VC-6), bexiga 62 (B-62), coração 7 (C-7), intestino grosso 4 (IG-4) e pericárdio 6 (CS-6), destacando-se os pontos locais vaso concepção 4 (VC-4) e vaso concepção 6 (VC-6). Os resultados se justificam em função dos efeitos analgésicos centrais da acupuntura, efeitos reflexos nos tecidos e alterações no fluxo sanguíneo. Da mesma forma, Wang, et al., (2019) utilizou pontos do meridiano do vaso concepção e percebeu uma melhora em relação a pacientes com síndrome de excesso (frio e umidade) e regulação benigna sobre a síntese de prostaglandina. Essa regulação na circulação sanguínea causa uma diluição de prostaglandinas intravasculares, bradicininas e histaminas, justificando o efeito analgésico.

Segundo Wang, et al., (2019) que utilizou os pontos bexiga 54 (B-54), estômago 28 (E-28), intestino grosso 4 (IG-4) entre outros, comparou a eficácia da acupuntura com o uso do medicamento Ibuprofeno. $\mathrm{O}$ resultado foi a diminuição significativa da intensidade da dor e uma baixa incidência de eventos adversos geralmente ocasionados com o analgésico convencional. O estudo de Li, et al., (2011), em que utilizou pontos semelhantes como o intestino grosso 4 (IG-4) e bexiga 54 (B-54), teve resultado semelhante com a diminuição do quadro álgico e o uso de medicamentos anti-inflamatórios não esteroides (AINEs): Ibuprofeno, Cetoprofeno, Naproxeno e Diclofenaco.

Segundo Wang, et al., (2019) que utilizou os pontos bexiga 54 (B-54), estômago 28 (E-28), intestino grosso 4 (IG-4) entre outros, comparou a eficácia da acupuntura com o uso do medicamento Ibuprofeno. O resultado foi a diminuição significativa da intensidade da dor e uma baixa incidência de eventos adversos geralmente ocasionados com o analgésico convencional. O estudo de Li, et al., (2011), em que utilizou pontos semelhantes como o intestino grosso 4 (IG-4) e bexiga 54 (B-54), teve resultado semelhante com a diminuição do quadro álgico e o uso de medicamentos anti-inflamatórios não esteroides (AINEs): Ibuprofeno, Cetoprofeno, Naproxeno e Diclofenaco.

Segundo Armour, et al., (2017), na MTC a dismenorreia é causada pelo fluxo irregular de Qi e sangue circulando pelos meridianos. Tanto Shetty B. et al., (2018), quanto Wang, et al., (2019) decorrem em seus achados que a acupuntura tem sido amplamente utilizada para tratar condições de dor e promover a livre circulação de Qi e sangue.

Já o estudo Sun, et al., (2018), utilizou pontos do meridiano do baço e pontos gatilho no mesmo trajeto desse canal. Ambos os grupos (controle e observação) tiveram o mesmo desfecho na sintomatologia e melhora da qualidade de vida das pacientes, porém o agulhamento e a indução do De Qi ao redor dos pontos regulares baço 6 (BP-6), baço 8 (BP-8) e baço 9 (BP-9) tiverem um efeito analgésico mais significativo do que o agulhamento dos pontos regulares. Wu et al. (2012) também abordou agulhamento em pontos gatilho na região do reto abdominal (borda lateral), ou seja, no trajeto do meridiano do baço. $\mathrm{O}$ resultado foi que $34 \%$ das mulheres $($ TrP-DN) tiveram reduções maiores $(\mathrm{P}<0,001)$ na dor do que aquelas que receberam 
placebo (32\%) ( $1^{\circ}$ mês: $\Delta-19,8 \mathrm{~mm}, 25,9$ a $-13,7 ; 2^{\circ}$ mês: $\Delta-20,1 \mathrm{~mm},-26,4$ a -13,8). Nesse estudo também se observou uma maior diminuição na quantidade de uso de medicamentos $(\mathrm{P}<0,001)$.

Pode-se considerar que Sun, et al., (2018) tratou as síndromes de obstrução por umidade-frio utilizando o ponto baço 8 (BP-8), como também a estagnação do Qi usando baço 6 (BP-6), Já Wu, et al., (2012) considerou pontos reflexos próximo aos pontos baço 14 (BP-14), baço 15 (BP-15) e baço 16 (BP-16) com a perspectiva de desencadear a liberação de encefalinas e endorfinas, promovendo assim o efeito analgésico.

E por fim, tanto Zhao, et al., (2017), quanto Wang et al. (2018) referiu a importância da indução do De Qi no processo de agulhamento do ponto baço 6 (BP-6), com a diminuição da intensidade da dor e do desconforto em mulheres com DP e síndrome de estagnação de frio e umidade. No entanto, ainda tem havido relativa discussão do fator potencial do De Qi na comunidade de pesquisa da acupuntura. Shi, et al., (2011) defendeu a tese de que é necessário a estimulação até a indução do De Qi e usou como exemplo a aplicação de agulhas nos pontos baço 6 (BP-6) e vesícula biliar 39 (VB-39), a fim de obter um maior efeito analgésico. Sendo assim, independentemente da quantidade de pontos utilizados é necessário que ocorra a indução do De Qi.

\section{Considerações Finais}

Os resultados levantados nesse estudo indicam que a acupuntura pode ser indicada para tratar mulheres com dismenorreia primaria por ser de baixo custo, não farmacológica e pouco invasiva. Para tal, vários pontos regulares de acupuntura foram utilizados e mostraram resultados satisfatórios.

Com base nos resultados obtidos, verificou-se que houve melhora significativa da dor, cólicas menstruais, dor de cabeça, tontura, diarreia, desmaios, cansaço, alterações de humor, vômitos e náuseas, demonstrando o efeito benéfico da acupuntura após o tratamento baseado em três ciclos menstruais, para mulheres com dismenorreia primária.

O tratamento da dismenorreia primaria com acupuntura, melhora a qualidade de vida e bem-estar da mulher que sofre desse desconforto, pois a acupuntura regula o equilíbrio do organismo, melhorando a circulação sanguínea, aumentando a resistência corpórea e sendo capaz de mudar a constituição corporal; por isso, reduz ao mínimo a necessidade de drogas e aumenta a eficácia terapêutica.

Os estudos também alertam para a necessidade do aumento das amostras e de tempo em estudos posteriores, pois por mais que a terapia com acupuntura possa ser capaz de diminuir a dor em pacientes com DP, os resultados precisam ser mais verificados devido ao pequeno tamanho das amostras.

\section{Referências}

Armour, M., Dahlen, H. G., Zhu, X., Farquhar, C., \& Smith, C. A. (2017). The role of treatment timing and mode of stimulation in the treatment of primary dysmenorrhea with acupuncture: An exploratory randomised controlled trial. PLOS ONE, 12(7), Artigo e0180177. https://doi.org/10.1371/journal.pone.0180177

$\mathrm{Bu}$, Y. Q. (2011) Observation on therapeutic effect of acupuncture at Shiqizhui (Extra) for primary dysmenorrhea at different time. Chinese Acupuncture \& Moxibustion,31(2), 110-2. https://pubmed.ncbi.nlm.nih.gov/21442807/

Bu, Y.-q., Du, G.-z., \& Chen, S.-z. (2011). Clinical study on the treatment of primary dysmenorrhea with preconditioning acupuncture. Chinese Journal of Integrative Medicine, 17(3), 224-227. https://doi.org/10.1007/s11655-011-0671-9

Chen, S. Z., Cong, Q., \& Zhang, B. F. (2011). Preliminary comparison on the time-effect rule of pain-relieving in the treatment of moderate dysmenorrhea between acupuncture on single-point and acupuncture on multi-point. Evid Based Complement Alternat Med,31(4), 3058. https://pubmed.ncbi.nlm.nih.gov/21528594/

Hang, Y., Zhou, Y., Guo, X., Liu, Y., Li, H., \& Chen, X. (2015). Analgesic effect on primary dysmenorrheal treated with conventional and sham acupuncture at San-yinjiao (SP 6). Chinese Journal of Basic Medicine in Traditional Chinese Medicine, 35(4), 318-22. https://en.Xmol.com/paper/article/1212898592878829570 
Hu, Y., Lin, Q., Li, Y., \& Zheng, X. (2011). Effect on PGF2alpha in plasma in primary dysmenorrhea treated with eye acupuncture. Journal of Traditional Chinese Medicine 2011, 31(8), 683-6. https://pubmed.ncbi.nlm.nih.gov/21894687/

Hu., N, Ma., L, Wang., P, Wu., G, Zhao., M, Hu., S, Sun., J, Wang., Y, Zhang., Z, Zhu., J, \& Ma., L. (2019). Influence of the quickness and duration of De Qi on the analgesic effect of acupuncture in primary dysmenorrhea patients with a cold and dampness stagnation pattern. Journal Traditional Chin Med, 39(2), 258-266. https://pubmed.ncbi.nlm.nih.gov/32186050/

Kiran, G., Gumusalan, Y., Ekerbicer, H. C., Kiran, H., Coskun, A., \& Arikan, D. C. (2013). A randomized pilot study of acupuncture treatment for primary dysmenorrhea. European Journal of Obstetrics \& Gynecology and Reproductive Biology, 169(2), 292-295. https://doi.org/10.1016/j.ejogrb.2013.02.016

Li, CH., Wang, YZ., \& Guo, XY. (2008). Acupuncture at Siguan points for treatment of primary dysmenorrhea. The Journal of Alternative and Complementary Medicine, 28(3), 187-90. https://pubmed.ncbi.nlm.nih.gov/18447217/

Li, YM., Bu, YQ., Hou, WJ., Chen, SZ., \&Gao, SZ. (2011). Observation on immediate analgesic effect of acupuncture at Shiqizhui (EX-B 8) only or multiacupoints in patients with dysmenorrhea: a randomized controlled trial. Complementary Therapies in Clinical Practice, 31(3), 199-202. https://pubmed.ncbi.nlm.nih.gov/21644298/

Lin, LL., Liu, CZ., \& Huang, BY. (2008). Clinical observation on treatment of primary dysmenorrhea with acupuncture and massage. Journal of Integrated Traditional and Western Medicine. Journal of Integrated Traditional and Western Medicine, 28(5), 418-20. https://pubmed.ncbi.nlm.nih.gov/18672768/

Liu, C.-Z., Xie, J.-P., Wang, L.-P., Liu, Y.-Q., Song, J.-S., Chen, Y.-Y., Shi, G.-X., Zhou, W., Gao, S.-Z., Li, S.-L., Xing, J.-M., Ma, L.-X., Wang, Y.-X., Zhu, J., \& Liu, J.-P. (2014). A Randomized Controlled Trial of Single Point Acupuncture in Primary Dysmenorrhea. Pain Medicine, 15(6), 910920. https://doi.org/10.1111/pme.12392

Liu, C.-Z., Xie, J.-P., Wang, L.-P., Zheng, Y.-Y., Ma, Z.-B., Yang, H., Chen, X., Shi, G.-X., Li, S.-L., Zhao, J.-P., Han, J.-X., Li, J.-D., Wang, Y.-X., Tang, L., Xue, X.-O., Li, M., Wang, Y., Sun, A.-p., Xing, J.-M., ... Liu, J.-P. (2011). Immediate Analgesia Effect of Single Point Acupuncture in Primary Dysmenorrhea: A Randomized Controlled Trial. Pain Medicine, 12(2), 300-307. https://doi.org/10.1111/j.1526-4637.2010.01017.x

Liu, Y.-q., Ma, L.-X., Xing, J.-m., Cao, H.-j., Wang, Y.-x., Tang, L., Li, M., Wang, Y., Liang, Y., Pu, L.-y., Yu, X.-m., Guo, L.-z., Jin, J.-1., Wang, Z., Ju, H.m., Jiang, Y.-m., Liu, J.-j., Yuan, H.-w., Li, C.-h., ... Zhu, J. (2013). Does Traditional Chinese Medicine Pattern Affect Acupoint Specific Effect? Analysis of Data from a Multicenter, Randomized, Controlled Trial for Primary Dysmenorrhea. The Journal of Alternative and Complementary Medicine, 19(1), 4349. https://doi.org/10.1089/acm.2011.0404

Ma, Y.-X., Ma, L.-X., Liu, X.-1., Ma, Y.-X., Lv, K., Wang, D., Liu, J.-p., Xing, J.-m., Cao, H.-j., Gao, S.-Z., \& Zhu, J. (2010). A Comparative Study on the Immediate Effects of Electroacupuncture at Sanyinjiao (SP6), Xuanzhong (GB39) and a Non-Meridian Point, on Menstrual Pain and Uterine Arterial Blood Flow, in Primary Dysmenorrhea Patients. Pain Medicine, 11(10), 1564-1575. https://doi.org/10.1111/j.1526-4637.2010.00949.x

Ma, Y.-X., Ye, X.-N., Liu, C.-Z., Cai, P.-Y., Li, Z.-F., Du, D.-Q., Guo, G., Chen, S.-Z., Zhao, J.-P., Liu, J.-J., Yi, H.-Q., \& Gao, S.-Z. (2013). A clinical trial of acupuncture about time-varying treatment and points selection in primary dysmenorrhea. Journal of Ethnopharmacology, 148(2), 498504. https://doi.org/10.1016/j.jep.2013.04.045

Pei, W., Peng, Z., Liangxiao, M., Hongwen, Y., Yuqi, L., Lin, C., Siyuan, X., Nijuan, H., Minyi, Z., \& Jiang, Z. (2016). Immediate analgesic effect of needling acupoints (bilateral De Qi vs unilateral De Qi ) on primary dysmenorrhea: a multi-center, randomized, controlled trail. Journal of Traditional Chinese Medicine, 36(6), 711-717. https://doi.org/10.1016/s0254-6272(17)30004-3

Ping, Huyou., Zhang, X., Zhang,LX., Wu, J., Wang, Z., Jia, XF., Tang, Y., \& Hui, RT. (2013). Time-effect study on instant analgesic effect of the dragon-tiger fighting needling method on primary dysmenorrhea. . J Tradit Chin Med, 33(11), 1005-9. https://pubmed.ncbi.nlm.nih.gov/24494291/

Ru, SQ., Zhang, P., Li, J., Wang, P., Lin, C., Hu, NJ., Hao, J., Hao, MY., Sun, JJ., Wang, YF., \& Zhu, J. (2016). Clinical Trials for Observing the Influence of Acupuncture Needle-stimulation Induced Sharp Pain on Curative Effect in Primary Dysmenorrhea Patients. Medicine Traditional, 41(2),154-8. https://pubmed.ncbi.nlm.nih.gov/27323444/

Shetty, G. B., Shetty, B., \& Mooventhan, A. (2018). Efficacy of Acupuncture in the Management of Primary Dysmenorrhea: A Randomized Controlled Trial. Journal of Acupuncture and Meridian Studies, 11(4), 153-158. https://doi.org/10.1016/j.jams.2018.04.001

Shulman, L. P. (2011). Effects of Acupuncture at Sanyinjiao (SP6) on Prostaglandin Levels in Primary Dysmenorrhea Patients. Yearbook of Obstetrics, Gynecology and Women's Health, 2011, 5-6. https://doi.org/10.1016/j.yobg.2011.06.048

Song, JS., Liu, YQ., Liu, CZ., Xie, JP., Ma, LX., Wang, LP., Zheng, YY., Ma, ZB., Yang, H., Chen, X., Shi, GX., Li, SL., Zhao, JP., Han, JX., Wang, YX., Liu, JP., \& Zhu, J. (2013). Cumulative analgesic effects of EA stimulation of sanyinjiao (SP 6) in primary dysmenorrhea patients: a multicenter randomized controlled clinical trial. The Cochrane Database of Systematic Reviews, 38(5), 393-8. https://pubmed.ncbi.nlm.nih.gov/24308187/

Sriprasert, I., Suerungruang, S., Athilarp, P., Matanasarawoot, A., \& Teekachunhatean, S. (2015). Efficacy of Acupuncture versus Combined Oral Contraceptive Pill in Treatment of Moderate-to-Severe Dysmenorrhea: A Randomized Controlled Trial. Evidence-Based Complementary and Alternative Medicine, 2015, 1-10. https://doi.org/10.1155/2015/735690

Sun, Y. N., Hou, X. S., Wu, J. Y., Tian, H. F., \& Zhao, J. P. (2018). Acupuncture Treatment of Primary Dysmenorrhea by Needling Acupoints of the Spleen Meridian with Positive Reactions : A Randomized Controlled Clinical Trial Acupuncture research, 43(5), 307-310. https://doi.org/10.13702/j.10000607.170989

Wade, C., Wang, L., Zhao, W. J., Cardini, F., Kronenberg, F., Gui, S. Q., Ying, Z., Zhao, N. Q., Chao, M. T., \& Yu, J. (2016). Acupuncture point injection treatment of primary dysmenorrhoea: a randomised, double blind, controlled study. BMJ Open, 6(1), Artigo e008166. https://doi.org/10.1136/bmjopen-2015008166 
Research, Society and Development, v. 10, n. 15, e346101522897, 2021

(CC BY 4.0) | ISSN 2525-3409 | DOI: http://dx.doi.org/10.33448/rsd-v10i15.22897

Wang, P., Zhang, P., Wu, GW., Hu, SQ., Li, J., Sun, JJ., Wang, YF., Zhao, MY., Hu, NJ., Zhu, J. (2018). Analgesic Effect of Deqi Induced by Needling at Sanyinjiao (SP 6) Acupoint on Primary Dysmenorrheal Patients with Cold Damp Stagnation Syndrome. Acupuncture research, 43(1):49-55. https://doi.org/10.13702/j.1000-0607.170310

Wang, H., Cao, Y., Jin, X., Yan, M., Wang, J., Li, R., \& Ji, L. (2019). Effect of an Acupuncture Technique of Penetrating through Zhibian (BL54) to Shuidao (ST28) with Long Needle for Pain Relief in Patients with Primary Dysmenorrhea: A Randomized Controlled Trial. Pain Research and Management, 19, 16. https://doi.org/10.1155/2019/7978180

Witt, C. M., Reinhold, T., Brinkhaus, B., Roll, S., Jena, S., \& Willich, S. N. (2008). Acupuncture in patients with dysmenorrhea: a randomized study on clinical effectiveness and cost-effectiveness in usual care. American Journal of Obstetrics and Gynecology, 198(2), 166.e1166.e8. https://doi.org/10.1016/j.ajog.2007.07.041

Wu, L. L., Su, C. H., \& Liu, C. F. (2012). Effects of Noninvasive Electroacupuncture at Hegu (LI4) and Sanyinjiao (SP6) Acupoints on Dysmenorrhea: A Randomized Controlled Trial. The Journal of Alternative and Complementary Medicine, 18(2), 137-142. https://doi.org/10.1089/acm.2010.0506

Xiong, J., Liu, F., Zhang, M.-m., Wang, W., \& Huang, G.-y. (2011). De-qi, not psychological factors, determines the therapeutic efficacy of acupuncture treatment for primary dysmenorrhea. Chinese Journal of Integrative Medicine, 18(1), 7-15. https://doi.org/10.1007/s11655-011-0857-1

Zhao, M.-y., Zhang, P., Li, J., Wang, L.-p., Zhou, W., Wang, Y.-x., She, Y.-f., Ma, L.-x., Wang, P., Hu, N.-j., Lin, C., Hu, S.-q., Wu, G.-w., Wang, Y.-f., Sun, J.-j., Jiang, S.-z., \& Zhu, J. (2017). Influence of de qi on the immediate analgesic effect of SP6 acupuncture in patients with primary dysmenorrhoea and cold and dampness stagnation: A multicentre randomised controlled trial. Acupuncture in Medicine, 35(5), 332-338. https://doi.org/10.1136/acupmed-2016011228

Zhi L. (2007). Randomised controlled study on superficial needling for treatment of primary dysmenorrhea. Chinese Acupuncture and Moxibustion, 27(1), 1821. https://pubmed.ncbi.nlm.nih.gov/17378197/ 